\title{
Flow Separation Associated with 3-D Shock-Boundary Layer Interaction (SBLI)
}

\author{
Rohan R. Morajkar ${ }^{1}$, Robin L. Klomparens ${ }^{1}$, W. Ethan Eagle ${ }^{2}$, James F. Driscoll ${ }^{3}$ and Mirko Gamba ${ }^{4}$ \\ University of Michigan, Ann Arbor
}

The first goal of this work was to determine the relative importance of two regions of flow separation that are caused by a shock wave boundary layer interaction (SBLI) in a rectangular duct. Separation occurs on both the bottom wall and the sidewall; the areas of these two separated regions were measured and compared. The location where separation first begins was also estimated. Most previous studies of SBLI have focused only on a single wall and have ignored interactions where walls meet at corners. A challenging problem that arose was how to best measure separation of 3-D flow that is sufficiently unsteady that the separation has become intermittent. This required that the probability of separation be measured. Two methods were applied to stereo-PIV velocity data in a SBLI occuring at a rectangular corner region of a Mach 2.75 wind tunnel. Method 1 is called the " $h$-criterion" and Method 2 is called the AVT (Axial velocity threshold) criterion. These methods are used to detect separation regions and provide a separation map at the interaction region. The importance of the side-wall separation regions versus the centerline separation is clearly evident from the results hence presented.

\section{Nomenclature}

$=$ principal flow direction

$=$ direction tangent to the bottom wall pointing leftwards looking downstream

$=$ direction perpendicular to $\mathrm{x}$ and $\mathrm{y}$ completing the right handed co-ordinate system

$=69.85 \mathrm{~mm}$, Height of the tunnel

$=57.2 \mathrm{~mm}$, Width of the tunnel

$=10 \mathrm{~mm}$ to $28.6 \mathrm{~mm}, \mathrm{y}$-span of the dataset considered for certain analysis

$=2.5 \mathrm{~mm}$ to $27 \mathrm{~mm}, \mathrm{z}$-span of the dataset considered for certain analysis

$=$ principal flow velocity or flow velocity in $\mathrm{x}$-direction

$=$ flow velocity in $\mathrm{y}$-direction

$=$ flow velocity in $\mathrm{z}$-direction

$=$ height of separation extending towards free stream at aparticular location

$=$ maximum height of separation in a particular image or separation bubble height

$=$ separation bubble starting location

$=$ number of images in a particular data plane

$=$ image no.

$=$ wall tangential direction

$=$ wall normal direction

$=99 \%$ boundary layer thickness $(\delta \approx 10 \mathrm{~mm}$ at the nominal interaction point $)$

\footnotetext{
${ }^{1}$ Graduate Student Research Assistant, Dept. of Aerospace Engineering.

${ }^{2}$ Post-doctoral consultant, Dept. of Aerospace Engineering, AIAA member.

${ }^{3}$ Arthur B. Modine Professor, Dept. of Aerospace Engineering, AIAA member.

${ }^{4}$ Assistant Professor, Dept. of Aerospace Engineering, AIAA member.
} 


\section{Introduction}

NE of the most interesting and not yet fully understood phenomenon in the area of high speed fluid dynamics is that of shockwave boundary layer interactions (SBLI). Such interactions are quite common in the external and internal aerospace applications and a detailed understanding of physics is quite necessary for accurate flow field and performance predictions of high speed air vehicles.

It is widely believed that the dynamics of shock-boundary layer interactions are primarily governed by the axial pressure forces arising due to the interaction of the adverse pressure gradient with the wall located on the streamwise plane of symmetry of the interaction zone. This face is essentially true for an inlet with relatively large aspect ratio, for which a two dimensional flow assumption is appropriate. However, this common assumption places more analytical importance on the separation occurring on this plane of symmetry than to the separation resulting from interaction with the side wall boundary layer. Yet experimental evidence of surface oil flow features contradicting this supposition [Reda, Murphy and Bruce Babinksy, Eagle et.al 2011] demonstrates qualitatively the effects of the side wall on the interaction dynamics of such a region are more prominent. This is especially important in low aspect ratio channels that resemble realistic air intake ducts more closely like that of a X-51 or concorde. What are missing are measurements, and statistical quantifications of these complex flow patterns and separation regimes in high speed devices which are essentially 3 dimensional.

In a steady, laminar flow, flow separation can be identified by using the oil film method or by using surface shear stress sensors. Separation is defined to occur where the shear stress at the wall is zero. As described by Surana et al.(2008), in a 3-D flow, it is the component of the shear stress vector that is perpendicular to the separation line that goes to zero at the wall; the component parallel to the separation line is not necessarily zero. If the flow is unsteady due to fairly intense turbulence, oil film and shear stress sensors are not as easy to interpret as their useful 2-D counterparts. Surface measurements also provide no information about the flow away from the wall, such as the height and shape of separation regions. Therefore in the present study we use instantaneous images of the velocity field near the walls that was obtained using stereo-PIV to provide a separation 'map' in the corner region where the flow is highly three dimensional.

\section{Previous Research}

There have been numerous studies of several simple "unit physics" SBLI problems; the reader is referred to comprehensive reviews written by Korkegi (1975), Adamson and Messiter (1980), Panaras (1996), Delery et al. (2010) and Babinsky and Harvey (2011). Relatively few previous studies have focused on 3-D unit physics problems, instead preferring to isolate the SBLI from any of three dimensional influences which turn out to have leading order effects on flow separation. Representations of the proposed flowfield resulting from a 3D interaction

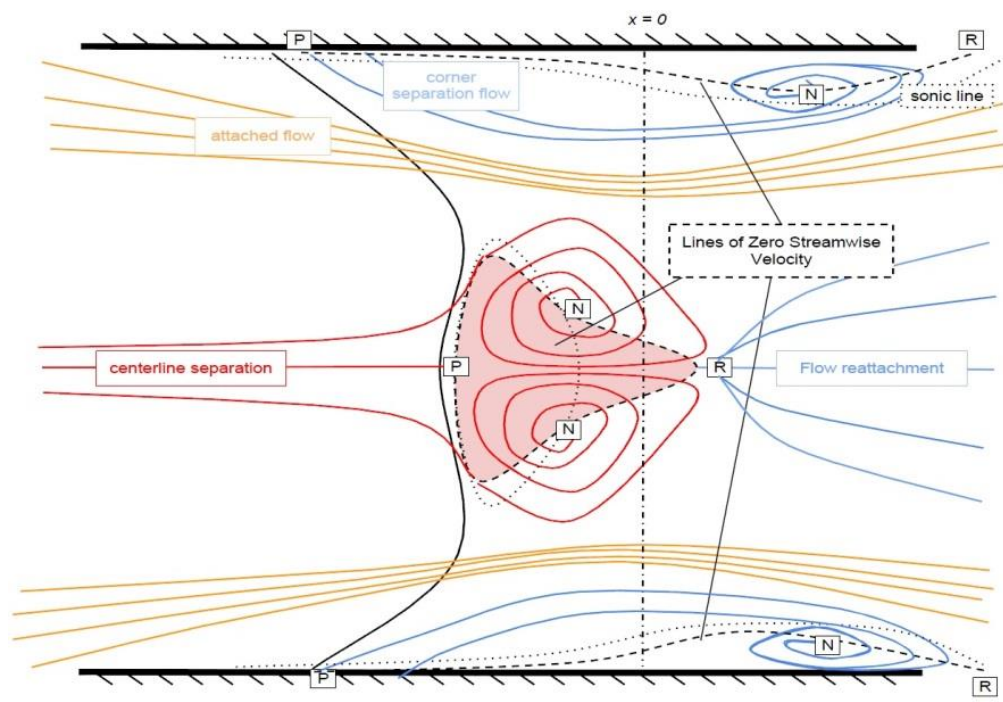

Figure 1. Schematic diagram(top view) of near bottom wall flowfield resulting from a 3-D shock /boundary layer interaction. Core flow direction: left to right. are shown in fig. 1 which shows the flow features developed in the interaction region formed due to oblique shock interaction formed on the bottom wall. The black curve represents the curved shock, which causes the flow to separate at the side wall(dark blue curves) as well as the bottom wall (red curves). Points $\mathrm{P}$ indicate points of flow separation, points $\mathrm{N}$ indicate the focus of the recirculation zone and points $\mathrm{R}$ are the points of flow reattachment. The side wall separation corresponds to the unit problem of swept shock boundary layer interaction (Alvi and Settles, 1992). The bottom wall separation corresponds to the unit physics proble of an oblique incident shock (Babinsky and Harvey, 2009).

Previous investigations of a SBLI in a rectangular duct are limited to two component PIV (Helmer et al. 2012) and several papers that report oil film and wall pressure data (Bruce and Babinsky 2010, Handa et al 2005, Doerffer \& Dallmann 1989). In their 
PIV work, Helmer et al. measured two velocity components on four different streamwise/wall-normal planes and all four of their planes were parallel. This was not sufficient to identify truly 3-D features and separation. Most recently, Eagle (2012) gathered stereo-PIV in an attempt to construct a comprehensive database inclusive of the three components of velocity along multiple orthogonal measurement planes in the three perpendicular directions. In this study we analyze some relavent data planes in this database to identify and investigate the regions of flow separation in the flow field, both on the bottom wall and side wall. The overarching objective is to develop a separation zone map in and around the interaction region along with quantified statistical parameters describing the unsteadiness of the separation.

\section{Experimental facilities}

The current experiments were performed in the low aspect ratio Michigan Glass Wind Tunnel (Lapsa and Dahm, 2011) which is a vacuum driven supersonic tunnel with a Mach 2.75 nozzle and a low aspect ratio $(57.2 \mathrm{~mm} \times 69.85 \mathrm{~mm})$ test section. This configuraton generates a three-dimensional inlet shock wave boundary layer interaction. The unit Reynolds's number for the experiment was $8.9 \times 10^{6} / \mathrm{m}$. A $6^{\circ}$ deflection angle, full span shock generator was used to produce an oblique shock wave. For the work and data presented

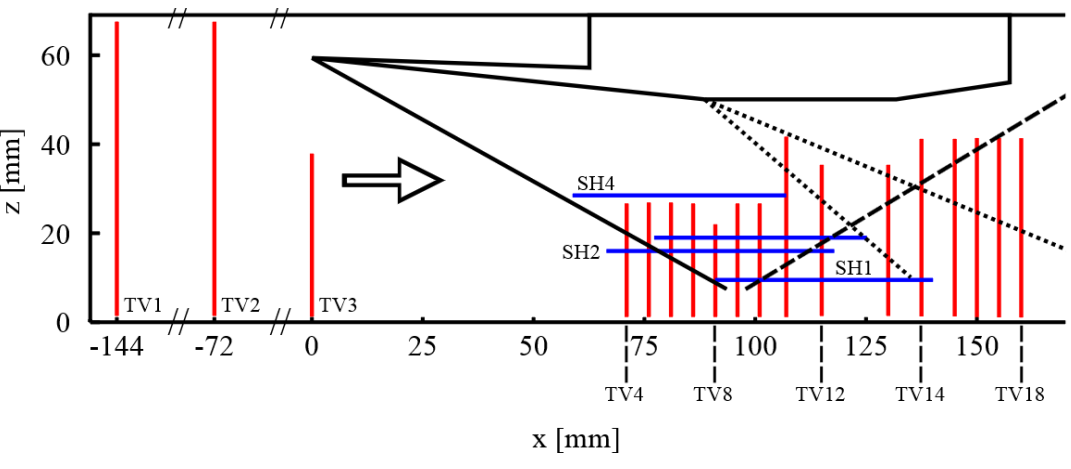

Figure 2. Schematic diagram(side view) of the tunnel showing various planes of measurement with respect to the wedge and incident shock.

here, the coordinate system being used is a right handed coordinate system with the $\mathrm{x}$-direction being the streamwise direction, $x=0$ being at the leading edge of the $6^{\circ}$ shock generator, and the z-direction being the wall-normal direction from the bottom wall. Following previous work on the investigation of our 3D SBLI system, (Eagle 2012; Eagle et al., 2012), further measurements were performed on four additional spanwise/wall-normal planes (Tranverse Vertical planes, TV) downstream of the primary interaction region. Only transverse vertical planes perpendicular to wall and the streamwise direction were considered for this analysis as they provide a better insight into area blocked by separation in a cross section of the tunnel. This selection was motivated with the aim of capturing the bottom wall and side wall separation around the interaction region. A summary of the location of all TV planes available is presented in Table 1, while a schematic of their orientation and location is shown in fig. 2.

Stereoscopic particle image velocimetry measurements were performed. Two interline transfer CCD cameras (resolution 1280x1024 pixel) in forward-scattering stereoscopic configuration were used for the imaging. To minimize particle drop-out while optimizing in plane displacement, the interframe time delay was set to $600 \mathrm{~ns}$ for all the measurements presented here. The double-pulse illumination of the flow was provided by a pair of low repetition rate, frequency-doubled Nd:YAG lasers timed with the pair of cameras by suitable timing units. Particle seeding of the flow was generated by a TDA-4B portable Laskin nozzle aerosol. The generator consists of an array of six Laskin nozzles that create polydispersed sub-micron particles using a Poly-Alpha Olefin (PAO) oil with density of $819 \mathrm{~kg} / \mathrm{m} 3$. The mean particle diameter is specified to be $0.281 \mu \mathrm{m}$. For a $\mathrm{M}=2.75$ free stream conditions of this study, the corresponding Stokes number is 0.025, which is within the acceptable range(Stoke's no. of 0.1) to track the large and small scale motion in the boundary layer and SBLI regions of interest as per Ragni et al ${ }^{[1]}$. The LaVision DaVis 7.2 and $\mathrm{DaVis} 8$ softwares were used for the acquisition of the measurement and processing of the data. The three-component velocity fields were reduced from the particle images using Davis 8. A multi-pass with reducing interrogation window size, window deformation and offset was used. The final size of the interrogation windows was $32 \times 32$ pixel, which corresponds to a projected physical size ranging from about $0.2 \mathrm{~mm} \times 0.2 \mathrm{~mm}$ to $0.7 \mathrm{~mm} \times 0.7 \mathrm{~mm}$ depending upon the measurement plane. A summary of the effective spatial resolution (measured as the size of the final interrogation window) for all planes considered here is given in Table 1 . 


\begin{tabular}{|c|c|c|c|c|c|}
\hline Plane & x [mm] & y [mm] & z [mm] & $\begin{array}{c}\text { Spatial } \\
\text { resolution } \\
\text { [mm x mm] }\end{array}$ & \# images \\
\hline TV5 & 76 & $1.17-35.06$ & $1.15-26.93$ & $0.21 \times 0.21$ & 402 \\
\hline TV6 & 81 & $1.17-35.06$ & $1.15-26.93$ & $0.21 \times 0.21$ & 402 \\
\hline TV7 & 86 & $1.17-35.06$ & $1.15-26.72$ & $0.21 \times 0.21$ & 402 \\
\hline TV8 & 91 & $1.17-35.06$ & $1.15-26.72$ & $0.21 \times 0.21$ & 402 \\
\hline TV9 & 96 & $1.17-35.06$ & $1.15-26.72$ & $0.21 \times 0.21$ & 402 \\
\hline TV10 & 101 & $1.61-44.92$ & $1.13-41.73$ & $0.34 \times 0.34$ & 1800 \\
\hline TV11 & 107 & $1.27-44.51$ & $1.37-35.39$ & $0.57 \times 0.57$ & 1100 \\
\hline TV12 & 115 & $1.44-44.75$ & $1.30-41.22$ & $0.67 \times 0.67$ & 1700 \\
\hline TV13 & 130 & $1.44-44.75$ & $1.30-41.22$ & $0.67 \times 0.67$ & 1800 \\
\hline
\end{tabular}

Table 1. Location and span of transverse vertical planes.

\section{Separation Criteria}

Two criteria were identified and applied to determine separation and its properties. In particular, separation was identified by using the following two criteria as applied by Souverein et al $(2010)^{[2]} \&$ Piponniau et $\mathrm{al}^{[3]}$ :

\section{A. Method 1: The "h-criterion"}

The separation height profile $\left(h_{k}(\mathrm{t})\right)$ of $\mathrm{k}^{\text {th }}$ image as a function of the surface location $\mathrm{t}$, is defined to be:

$$
\int_{0}^{h_{k}(\mathrm{t})} u_{k}(\mathrm{t}, \mathrm{n}) \cdot d \mathrm{n}=0
$$

where $t$ is the direction tangent to the wall, $n$ is the direction normal to the wall (i.e., $t$ ) and $u_{k}(t, n)$ is the local streamwise velocity at the $(t, n)$ point. The quantitiy $h$ is computed for each measurement instant $k$ and it is denoted by $h_{k}$. By definition, if separation is not detected, $h_{k}=0$. The ensemble average quantity, here denoted by $h$, is computed by averaging the set of local values $h_{k}$. This parameter effectively tells us how much of wall normal space is effectively reduced because of separation. This is equivalent to that region being unavailable to the incoming flow but it must be noted that the current measurments don't include the effect of density. This quantity is limited by the accuracy of velocity measurements near the wall and by the overall spatial resolution of the measurements. As a result, vector validation, interpolation and low-pass filtering was performed as described below.

From each profile instantaneous $h_{k}$ profile, the maximum value of the profile is extracted and denoted by $H_{k}$. This quantity is simply referred to as separation bubble height and provides a global instantaneous measure of the wall-normal extent of separation. Unlike the quantity $h_{k}$ which is a function of one coordinate direction, $H_{k}$ is a constant for a particular image. The ensemble average separation bubble height $H$ is then computed from the set of instantaneous values.

\section{B. Method 2: The AVT (Axial Velocity Threshold) criterion:}

The probability of separated flow at each point in the measurement domain was defined and computed as the fraction of the total number of realization where the local instantaneous streamwise velocity was less than a threshold value, here taken as $20 \mathrm{~m} / \mathrm{s}$. The quantity thus computed represents the probability of the local velocity to be less than the threshold value at a particular location in a data plane. A threshold value of $20 \mathrm{~m} / \mathrm{s}$ is chosen to

4

American Institute of Aeronautics and Astronautics 
make the method resistant to errors arising from the PIV dynamic resolution. As a consequence, this definition identifies regions where both reverse and low-velocity regions exist. Thus, it tends to overestimate the distribution and size of the region where reverse flow might exist. In any case, this definition is used to identify the regions that are most affected by the shock interaction process and we loosely refer to them as regions of probable separation.

All points with a probability greater than $50 \%$ were said to lie near a separated zone. The isoconotur line of $50 \%$ probability, denoted as $h_{A V T}$, was chosen as the dividing line between disturbed and undisturbed flow . The area under the dividing line $h_{A V T}$ can be understood as the separation or blockage area similar to $h_{k}$. This definition of separated flow is more robust than the corresponding one that could be defined from the separation profile $h$ because it is less prone to the experimental limitations near the wall as a low velocity region resulting from separation can extend towards the core flow away from the wall, where the accuracy of PIV is sufficent. However, it incorporates an arbitrary parameter (50\% intermittency) that hinders identification of highly unsteady separation. To address this latter issue, different threshold values were considered and similar conclusion were made with the value shown here.

\section{Results}

In order to detect separation, the above metrics and methods were applied to transverse/vertical measurement planes around the interaction zone (TV5 to TV13 planes). The data was pre-processed using PIVMAT, a PIV toolbox for MATLAB developed by F. Moisy ${ }^{[4]}$. All the data for the $h$-criterion analysis was filtered using the smoothing and interpolating method developed by $\mathrm{D}$. Garcia ${ }^{[5]}$, which is based on a penalized least square regression. A smoothing parameter value of 1.25 was used. In case of TV planes the corner region from $(y=0, z=$ $0)$ to $(y=10 \mathrm{~mm}, \mathrm{z}=2.5 \mathrm{~mm})$ has been deleted from statistical analyses to avoid the mathematical complexity of defining the separation area as the corner region was approached ( $h$-criterion). However, the corner region has been considered in analyses of $h_{A V T}$. The incident shock wave impinges on the bottom wall boundary layer at $\mathrm{x}=95 \mathrm{~mm}$ on the centerplane of the tunnel $(\mathrm{y}=28.6 \mathrm{~mm})$. Owing to curvature of shock, the location of incident shock impingement moves upstream as we move off centerline. Results corresponding to two representative planes, one upstream (TV5) and one downstream (TV11) of the interaction zone are presented below to demonstrate the spatial distribution of the separated flow region.

\section{A. $h$-criterion}

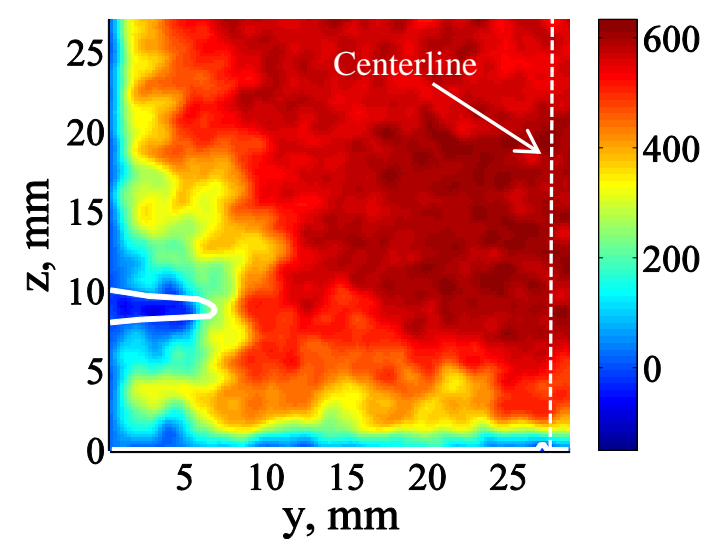

Figure 3. A single image from TV5 $(x=76 \mathrm{~mm})$ dataset showing the separation profile, $h_{\mathrm{k}}$, superimposed over instantaneous streamwise velocity $(\mathrm{m} / \mathrm{s})$

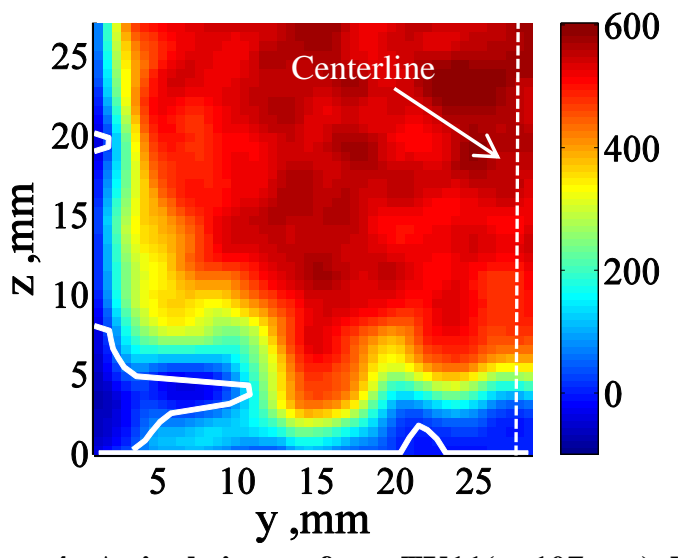

Figure 4. A single image from TV11 $(x=107 \mathrm{~mm})$ dataset showing the separation profile, $h_{\mathrm{k}}$, superimposed over instantaneous streamwise velocity $(\mathrm{m} / \mathrm{s})$ contours

Fig. 3 and fig. 4 show a representative instantaneous view of the separation profile $h_{k}$ (white solid line) superimposed to the streamwise (out-of-plane) velocity color contour for a transvserse/vertical plane at $76 \mathrm{~mm}$ (TV5, before the incident shock impingement) and $107 \mathrm{~mm}$ (TV11, after the incident shock impingement). Using the definition of equation (1) the profile $h$ was computed for the bottom-wall according to:

And for the side-wall according to:

$$
\int_{0}^{h_{k}(\mathrm{y})} u_{k}(\mathrm{y}, \mathrm{z}) \cdot d \mathrm{z}=0
$$

$$
\int_{0}^{h_{k}(\mathrm{z})} u_{k}(\mathrm{y}, \mathrm{z}) \cdot d \mathrm{y}=0
$$


We define separation to pertain to side-wall if it occurrs below $\mathrm{y}<10 \mathrm{~mm}$ otherwise it is attributed to the bottom wall. The total area under the $h_{k}$ profile is refered to as the total separation area.

The first observation to be made from the examples of Fig. 3 and 4 is that the side-wall separation is larger than the bottom-wall separation and it is most prominent near the corner of the tunnel. Plane TV5 (Fig. 3) is located at 76 $\mathrm{mm}$, which corresponds to about $2 \delta$ upstream of the centerline interaction ( $\delta$ being the boundary layer thickness at the interaction point for the undisturbed flow). At this location and instant, the side-wall separation close to the corner is already developed while the bottom-wall boundary layer near the centerplane of the tunnel $(28 \mathrm{~mm})$ isn't strongly influenced by the incident shock. It is apparent from comparing Figs 3 and 4 that the side-wall separation region in TV11 stretches further into the free stream region than TV5 indicating growth of the separation through the SBLI.

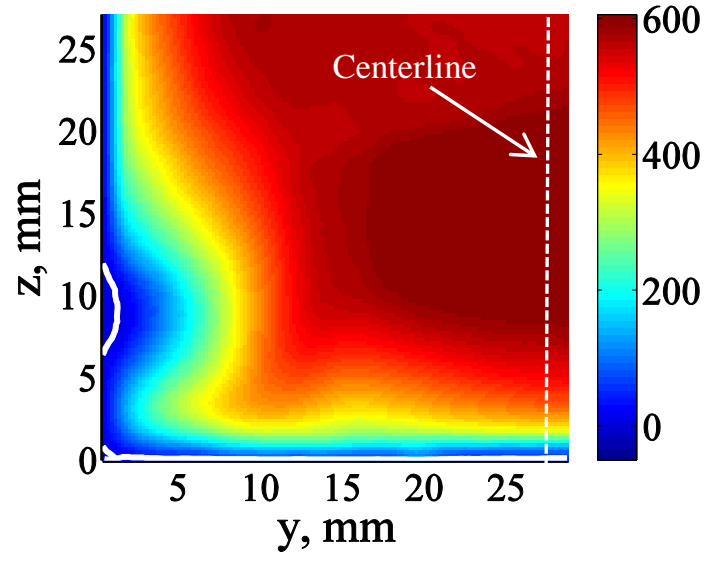

Figure 5. Mean separation profile superimposed over mean streamwise velocity in TV5 plane $(x=76 \mathrm{~mm})$ computed using h-criterion

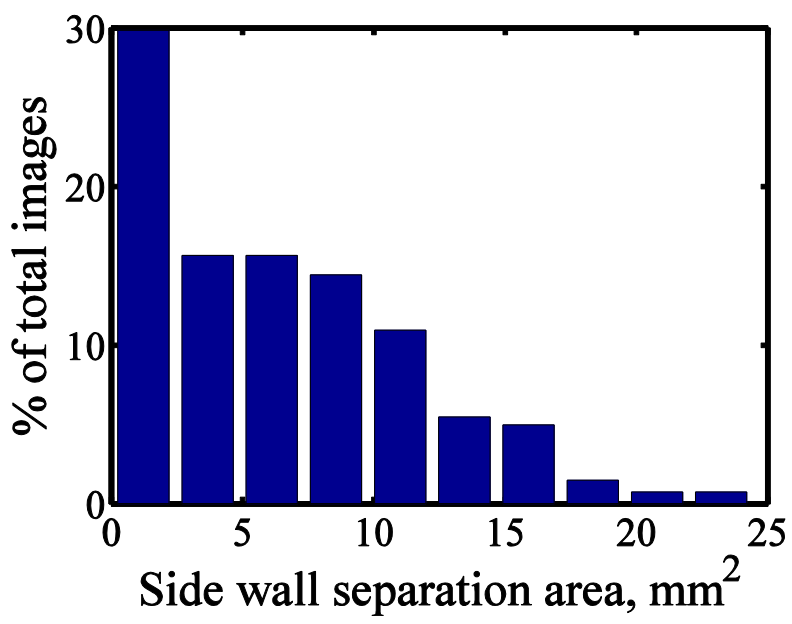

Figure 7. Histogram of side wall separation area in TV5 plane $(x=76 \mathrm{~mm})$

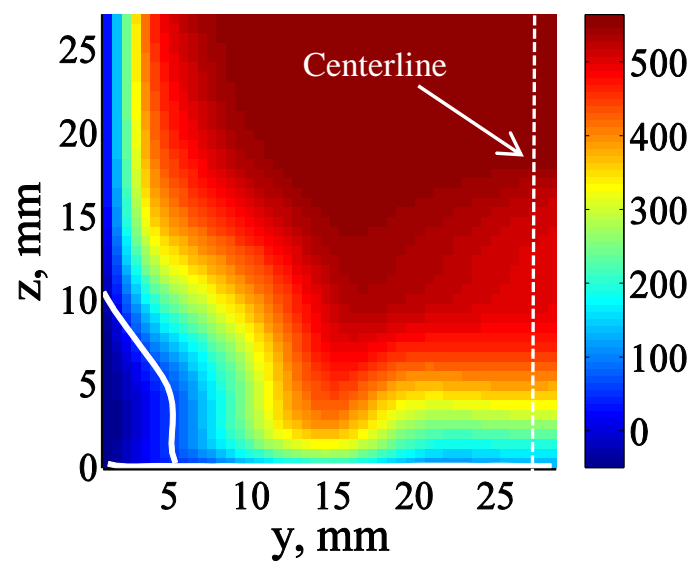

Figure 6. Mean separation profile superimposed over mean streamwise velocity in TV11 plane $(x=107 \mathrm{~mm})$ computed using h-criterion



Figure 8. Histogram of side wall separation area in TV11 plane $(x=107 \mathrm{~mm})$

The ensemble average separation profiles $h$ corresponding to the planes of Figs. 3 and 4 are shown in Figs. 5 and 6 . The quantity $h(y)$ represents the mean bottom-wall separation while $h(z)$ represents the mean side wall-separation profile. The area under these curves is a representation of the region in the wind tunnel where there is no net flow on 
average. Comparing Figs. 5 and 6, flow blockage is stronger in the TV11, which is downstream of the interaction than in TV5 plane. Also, most of the contribution to the blockage comes from the side-wall separation in both planes. The bottom wall separation is nearly non-existant in the mean at these two specific locations only appearing in the immediate vicinity of the predicted centerline interaction. This behavior is ascribed to the significant threedimensional effects arising due to the low aspect ratio wind tunnel and the complex shock structure of the incident shock as the corner is approached (Helmer et al, 2012) ${ }^{[6]}$.

Figures 7 and 8 show the probability density function (PDF) distribution of side-wall separation area compiled over all instants for the TV5 and TV11 planes, respectively. The upstream plane (TV5) has a smaller side-wall separation and it is more intermittent than side-wall separation at the downstream location (TV11). The PDF of fig. 7 shows that the most probable flow state at TV5 is attached flow. However, note that the finite resolution of the measurement and practical limitations in measuring the flow velocity as the wall is approached could also be responsible for the lack of separation detection.

Further downstream the most probable value of the side-wall separation area in TV11 plane is about $27.5 \mathrm{~mm}^{2}$ and there is no case of non-separated flow as seen in fig. 8. For comparison, assuming that separation is symmetric relative to the centerplane, quarter of the windtunnel corresponds to $1000 \mathrm{~mm}^{2}$. Therefore, the fractional area of separation can be a significant fraction of the available channel area; at the most, the side-wall separation on the TV11 plane amounts up to $6 \%$ of the quarter area.

PDF distributions of the bottom-wall separation area for the TV5 and TV11 planes are shown in figs. 9 and 10, respectively. It is evident that the bottom-wall separation is more probable at the upstream location of TV5, although at both locations the predominant state is attached flow. The proposition by J. Délery in the book by Babinsky \& Harvey ${ }^{[7]}$ which may explain this behavior is that the incident shock impinges on the flow around separation bubble, which acts as a free boundary causing an expansion wave to reflect off in the downstream direction. The expansion wave curves the detached shear layer towards the wall eventually reattaching it downstream of the interaction. At the same time, the influence of high pressure developed behind the shock is propagated upstream through the viscous boundary layer. This smears the adverse pressure gradient upstream of the point where the shock impinges the boundary layer on the bottom wall which causes a tendency for the flow to separate. In Figs. 7 through 10 we show that most of the flow separation in our low aspect ratio supersonic duct 3D SBLI comes from the interaction of the shock with the side-wall boundary layer and not from interaction with the bottom-wall.

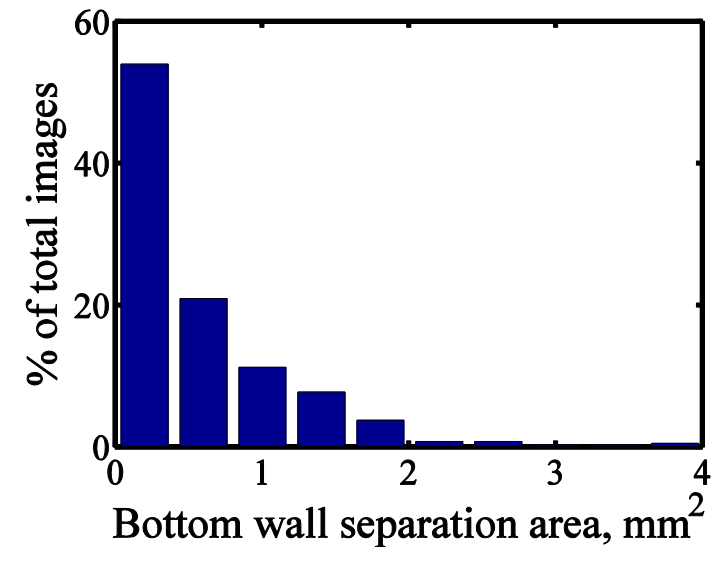

Figure 9. Histogram of bottom wall separation area in TV5 plane $(x=76 \mathrm{~mm})$

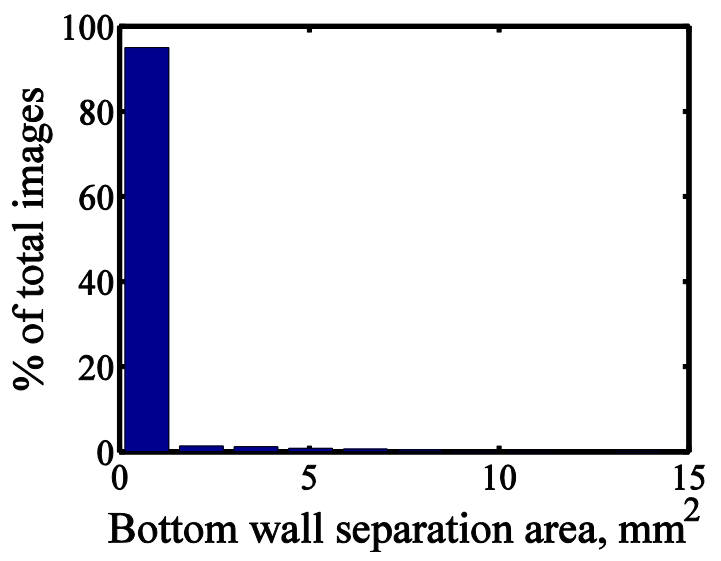

Figure 10. Histogram of bottom wall separation area in TV11 plane $(x=107 \mathrm{~mm})$ 


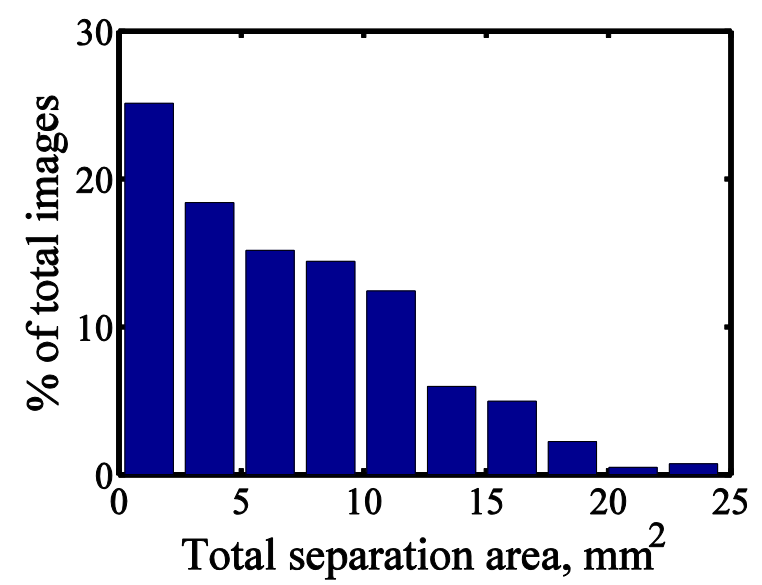

Figure 11. Histogram of total separation area in TV5 plane $(x=76 \mathrm{~mm})$

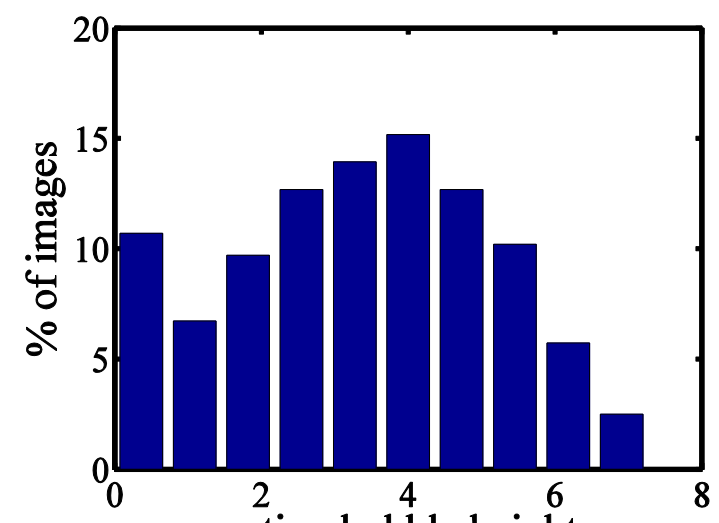

separation bubble height, $\mathrm{mm}$

Figure 13. Histogram of side wall separation bubble height in TV5 plane $(x=76 \mathrm{~mm})$

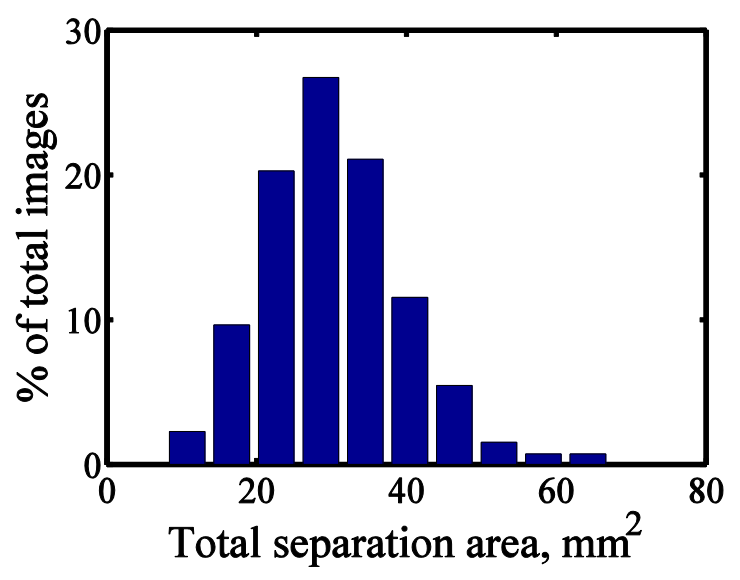

Figure 12. Histogram of total separation area in TV11 plane $(x=107 \mathrm{~mm})$

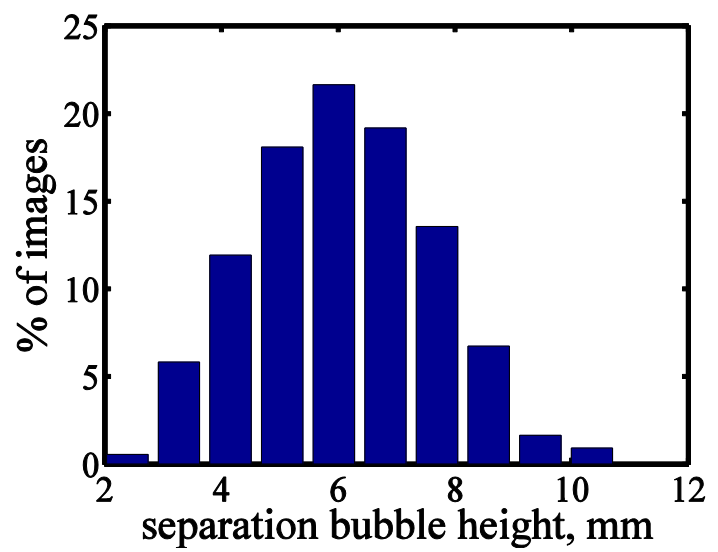

Figure 14. Histogram of side wall separation bubble height in TV11 plane $(x=107 \mathrm{~mm})$

PDF distributons computed for the total separation area from all the images of the TV5 and TV 11 planes are shown in fig. 11 and fig. 12, respectively. For the most part, these PDF distributions follow the profiles for the corresponding side-wall separation areas. Separation is more probable and stronger in the region downstream of the interaction than the upstream plane.

Figures 13 and 14 show the PDF distribution of side-wall separation bubble height $H_{k}$ for the two planes considered here. This quantity represents how far the separation profile stretches into the undisturbed flow. A value of $H_{k}$ equal to $0 \mathrm{~mm}$ corresponds to a non-separated case. . The peak at separation bubble height of $0 \mathrm{~mm}$ in TV5 which is an outlier to the bell curve profile may be the result of lack of PIV resolution near wall and the way separation profile $h_{k}$ is defined in that case. It is worth noting that past the interaction zone, the separation bubble is more likely to stretch towards the core flow than in the upstream region. On the upstream plane, separation bubble height extends up to $1 / 2 \delta$, whereas on the downstream plane it reaches one boundary layer thickness. PDF distributions of the bottom-wall separation bubble height were also compiled and they further indicated that the the flow is hardly separated at the location of the TV11 plane but it is weakly separated at TV5. 


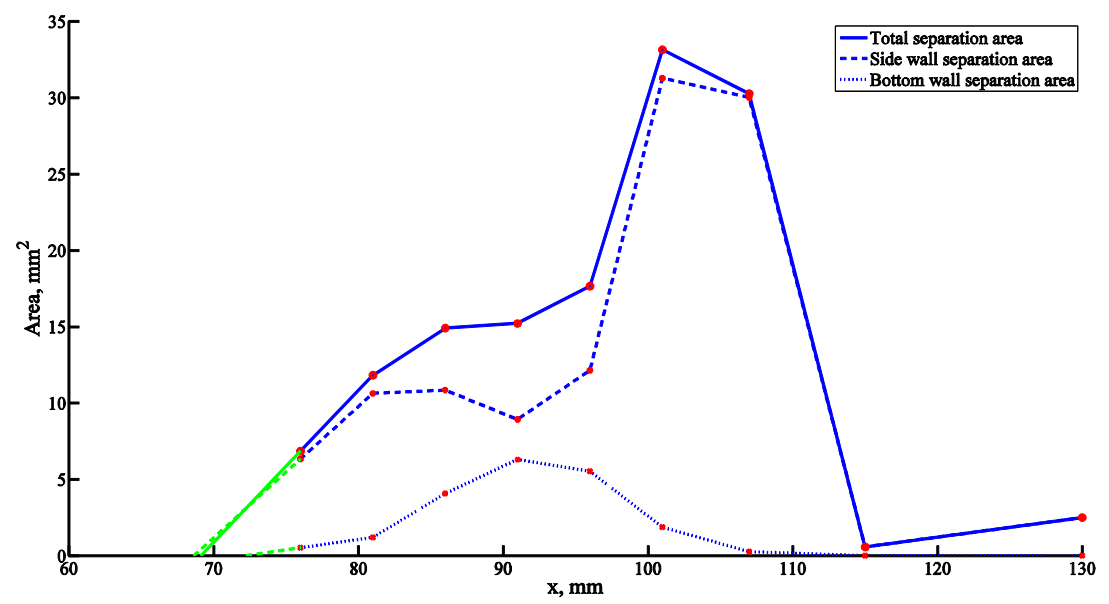

Figure 15. Mean Separation area vs $x$ as computed using true separation profile (green lines are the linear extrapolation of the data)

The $h$-criterion analysis presented above was repeated for the planes around the interaction region to obtain the variation of separation properties with respect to the streamwise direction $x$. The streamwise variations of the ensemble averaged total, bottom- and side-wall separation areas (computed over all instances) are shown in Fig. 15. Since the relevant measurements started after a streamwise location of $70 \mathrm{~mm}$, the data were linearly extrapolated to zero (green lines) to obtain the upstream point of separation, which is found to be around $x=70 \mathrm{~mm}$.

It can be seen from the graph in Fig. 15 that most of the contribution to the total separation area comes from the side-wall separation as compared to that from bottom-wall separation zone in almost all the streamwise locations around the nominal interaction location $(x=95 \mathrm{~mm})$. This is particularly true past the nominal interaction location where all of the separated area is due to side-wall separation. The bottom-wall separation grows from $\mathrm{x}=70 \mathrm{~mm}$, peaks around the nominal interaction location $(x=95 \mathrm{~mm})$ and then decreases to zero immediately after the interaction. On the other hand, the side-wall separation increases above the bottom-wall separation in the region leading to the nominal interaction region, drops at a local minimum value around the nominal interaction region, and then further increases (and dominates) past the nominal interaction location. At the nominal interaction location, where the bottom wall separation is the strongest, the bottom-wall separation area accounts for about $35 \%$ of the total separation area. The authors note that this result could impact the placement and testing of various boundary layer control devices, which have typically been installed only on the bottom walls of test inlet geometries.

The dominance of the side-wall separation is attributed to the highly three-dimensionality of the flow and of the SBLI. In the region just before the nominal interaction location, the bottom-wall separation zone strengthens due to the adverse pressure gradient from the shock felt upstream, which may cause the side-wall separation zone to weaken (relative to the incoming state) in the same zone; nevertheless, the contribution of side-wall separation to the total separation remains greater than the contribution of bottom-wall separation. After the nominal interaction location, the bottom-wall separation zone reattaches due to the expansion fan reflected from the incident shock. However, at this position, the 3D effects of the interaction resulting from the low aspect ratio of the tunnel dominate. As the centerline interaction is diminishing the side-wall boundary layer separation continues to grow.The increasing of one separation strength and weakening of the other seemed to indicate existence some connection between side-wall and bottom-wall separation areas. Analysis of the separation size among instantaneous images did not yield any correlation between sizes. Another behavior worth noticing from the green exterpolated curves is that the side wall separation bubble seems to start much upstream at around $\mathrm{x}=68 \mathrm{~mm}$ of the central interaction $(\mathrm{x}=$ $95 \mathrm{~mm}$ ) whereas the bottom wall separation bubble starts at around $\mathrm{x}=72 \mathrm{~mm}$. It is postulated that this may be caused by the curved shape of the shock near the side-walls along with the viscous side wall boundary layer carrying the effect of pressure rise due to this curved shock further upstream resulting in adverse pressure gradient even on the side wall causing separation further upstream.

Plots for the streamwise variation of the ensemble average separation bubble height $H$ defined from the set of instantaneous values showed a behavior similar to that of fig. 15 with approximately similar start points. 


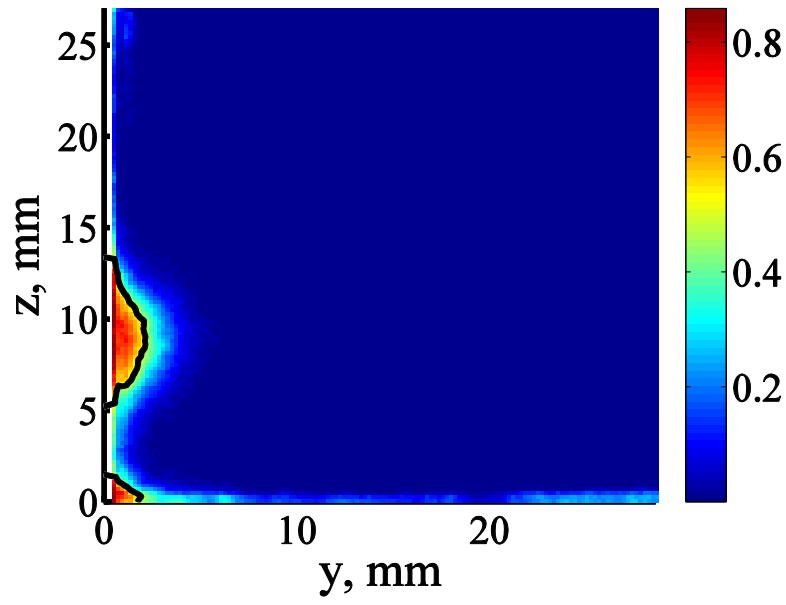

Figure 16. Probable separation region outline $\left(\boldsymbol{h}_{\mathrm{AVT}}\right)$ superimposed on the mean intermittency map of TV5 $(\mathrm{x}=76 \mathrm{~mm})$

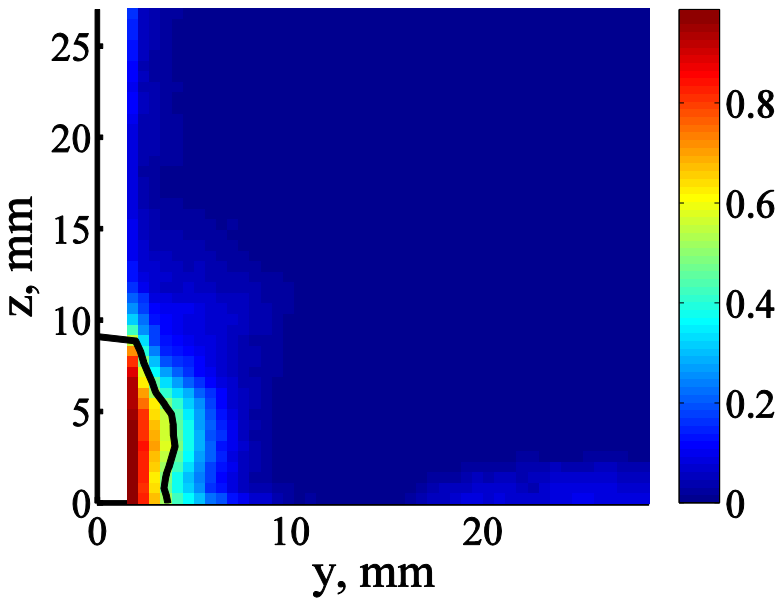

Figure 17. Probable separation region outline $\left(\boldsymbol{h}_{\mathrm{AVT}}\right)$ superimposed on the mean intermittency map of TV11 $(x=107 \mathrm{~mm})$

\section{B. Probability of separated flow}

The separation profile $h_{A V T}$ (black solid line) superimposed to the probability of separation (defined based on the streamwise velocity being less than $20 \mathrm{~m} / \mathrm{s}$ ) for the TV5 and TV 11 planes, is shown in Fig.s 16 and 17 respectively. The separation profile $h_{A V T}$ was defined as the isocontour of $50 \%$ probability to observe a streamwise velocity below the threshold. The outline of the separation profile extracted from the intermittency map of separated flow is qualitatively similar to that obtained by the $h$-criterion (Figs. 5 and 6). The region between the walls (axes) and the separation profiles represent the zone along the wall where the flow is most likely to be separated. Greater the distance of this profile from the wall, higher the probability of separation at that location. The bottom-wall separation which was detected using the $h$-criterion, is not detected using this method since the probability of flow to be separated on the bottom wall is nearly absent in TV11 and is well below 50\% on TV5.

The area under the separation profile $h_{A V T}$ gives a measure of likeliness of the flow to be separated. Similarly to the other method, the area of separted flow was computed for all available transverse/vertical planes and it is plotted as a function of streamwise direction in Fig. 18. The green line is a linear extrapolation of the available data to zero. The area of separted flow of Fig. 18 can also be interpreted as an overall probability of separation at a particular $x$ location (i.e., on the particular measurement plane) due to the way it is defined. It can be seen that the probability of separation on the bottom wall is 0 upstream of about $x=81 \mathrm{~mm}$, it then increases and peaks around $x=90 \mathrm{~mm}$ due to the adverse pressure gradients imposed by the shock which impinges on the boundary layer downstream at about $x=95 \mathrm{~mm}$ on the horizontal streamwise centerline of the tunnel. The area of separated flow then decreases and the flow reattaches near $x=107 \mathrm{~mm}$. Similar behavior can be observed for side-wall and total separation. The side-wall separation begins upstream of the location where bottom-wall separation is observed first, which is consistent with the behavior extracted from the $\mathrm{h}$-critereon analysis. The results corresponding to the side wall separation start point seem to match fairly well with those obtained from the $h$-criterion. Note that this method shows a higher probability of flow to be separated at the bottom wall than the side wall at around $x=90 \mathrm{~mm}$.

Since the $h$-criterion method computes the separation area by averaging over all the events, it will be highly biased towards the most probable value in case of distributions with lower variance (note that the spread of the histogram of bottom wall separated area is thinner that that of the side wall separated area), which means that the value of the average separation area is biased towards 0 at locations well upstream of the nominal interaction point. On the other hand, the AVT method essentially filters out regions of low probability of separation because a probability value of $50 \%$ is used in the analysis; thus, this second method identifies the bottom wall separation start point to be located further downstream than that calculated from the $h$-criterion method if the flow is less than $50 \%$ separated. 




Figure 18. Separation area vs $\mathrm{x}$ as computed from the separation profiles characterized by regions showing $50 \%$ probability of having streamwise velocities less than $20 \mathrm{~m} / \mathrm{s}$ (green lines are the linear exterpolation of the data)

\section{Three-dimensional view of regions of probable separation}

A three-dimensional view of the system of interests and of the regions of most probably flow separation is shown in Fig. 19. The figure is a compilation of the probability of separated flow for each of the transverse/vertical planes located around the nominal shock interaction region $(x=95 \mathrm{~mm})$. The figure also includes a schematic representation of the inviscid incident and reflected shock waves (green semi-transparent surface) for reference only, and a representation of the compression surface generating the incident shock on the top surface of the windtunnel. Only one half of the windtunnel span is shown for clarity. The contours in the figure identify the regions where the flow is more likely to separate. The bottom wall separation occurs only around the nominal interaction region, while the side-wall separation begins well upstream and persists downstream. Because of the low-aspect ratio of the tunnel, the incident shock is strongly deformed, and the impact of the side-wall boundary layer, and the dynamics of the 'fin-wall' interaction appears to dominate the flowfield. Furthermore, separation remains confined near the corner, and the geometric center of the area of probable separation does not vary much from the bottom wall-normal height of about $10 \mathrm{~mm}$.

In our configuration (but in reality in any SBLI configuration), we can identify two separate SBLI events: near the centerplane of the windtunnel, where the SBLI resembles the classical two-dimensional incident shock interaction, and near the side wall, where a sharp-fin SBLI is propagating towards the corner. The proposed structure of the three-dimensional interaction that result in bottom- and side-wall interaction is shown in detail in Figure 20. The structure shown in the figure is inferred from combining the general structure of the 2-D incident shock interaction and the structure of a fin-induced swept shock interaction as proposed by Alvi and Settles (1992) ${ }^{[13]}$. Because of the importance of the side-wall separation, we will here focus on the swept-shock interaction component.

In the swept shock interaction configuration, the strength of the interaction is governed by the component of the Mach number of the incoming flow normal to the invisicid shock wave $\left(M_{n}\right)$. For sufficiently large values of $M_{n}$, the flow separates, and different separation structures are observed based on the strength of the interaction $\left(M_{n}\right)$. For weak interactions, a single separation region is observed (this is the case indicated in Figure 20), while a second separation region arises for stronger interactions. One peculiar characteristic of swept shock interaction is its quasiconical symmetry with the origin of the conical flow located somewhat upstream of the leading edge of the fin. As a result, the flow field in a conical system of reference $(\theta, \phi)$ is self-similar. The general flow structure that evolves around the region of flow separation consists of bifurcated $\lambda$-shock originated by the invisicid shock wave which induces flow separation. A forward and rearward separation shock waves are generated around the separation region. A slip line originates from the triple point of the $\lambda$-shock. For the case of this study, $M_{n} \approx 1.2$. This case corresponds to a relatively weak interaction that results in incipient separation ${ }^{[18]}$. Thus, only a single separation region might be expected ${ }^{[13]}$. In Figure 20, the separation is bounded by the separation point $\mathrm{S}$ and reattachment point A. Point $U$ is the upstream point of influence. The region bounded by the slip line and the outer boundary of the 
separation region defines what is referred to as "impinging jet". In this region, the flow curves around the separation

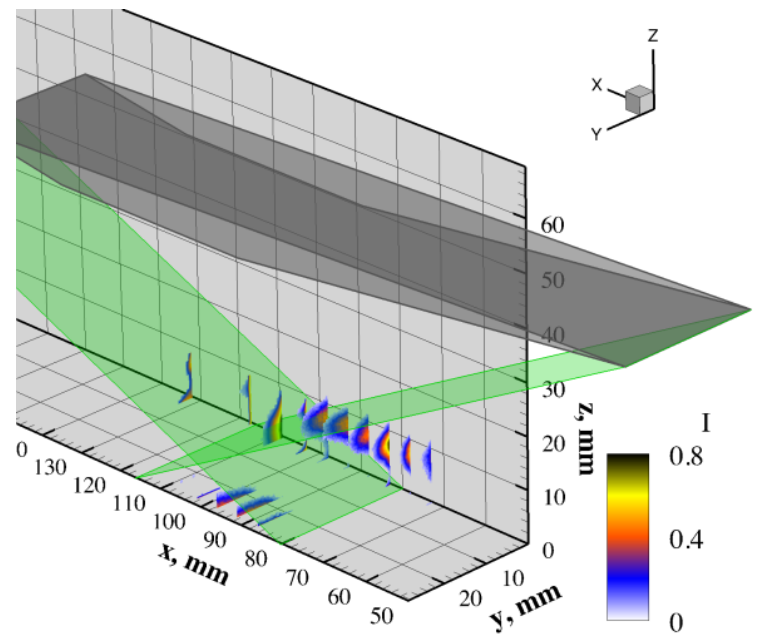

Figure 19. 3D view of the regions of probable separation. Green planes is a qualitative representation of the invisicid incident and reflected shock.

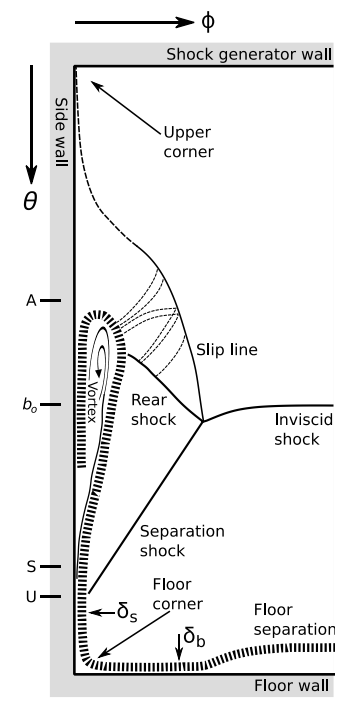

Figure 20. Schematic diagram (front view) of near side wall flowfield resulting from a 3-D shock /boundary layer interaction. Core flow direction: into the plane. Half channel width is shown.

bubble through a set of expansion waves. The separation bubble is dominated by a vortex

existing in the core of the separation region. The results of the current study are consistent with the view of Figure 20 and identify the region where separation is more likely. Because PIV measurements are taken on planes perpendicular to the streamwise flow direction and not on planes that capture the conical symmetry of the flow, it is difficult at this time to describe the flowfield using this conical frame of reference. Despite the progress here-in, more work is needed to assess these aspects of side-wall separation, its structure, and its dependence and relation to the bottom-wall separation.

\section{Conclusion}

From the analysis conducted, it can be concluded that the side wall separation is stronger than bottom wall separation in the three-dimensional SBLI in a low aspect ratio duct. Our results indicate the side wall separated cross sectional area averages 2-3 times larger than the bottom wall. Also the flow separation is less intermittent (more likely to be separated) on the side wall than on the bottom wall. And the curved nature of shock in such an interaction causes the flow to separate on the side wall upstream of where it begins to separate on the bottom wall.

Both the $h$-criteron and the ATV methods produced similar results for the separation point and separated area. The axial velocity threshold method was better at filtering out noise in the PIV data but filtered out the low probability events, while the $h$-critereon was sensitive to noise arising from experimental limitations. On the other hand the h-critereon method made it possible to obtain a detailed picture about the statistical properties of the separation at a particular location.

\section{Acknowledgments}

This work is sponsored by the US Air Force Collaborative Center for Aeronautical Sciences, which is supported by the Air Force Research Laboratory, Air Vehicle Directorate, AFRL/RB and monitored by Dr. John (Jack) Benek, Jon Tinapple, and Lewis Surber.

\section{References}

${ }^{1}$ D. Ragni, F. Schrijer, B. W. van Oudheusden and F. Scarano, "Particle tracer response across shocks measured by PIV", Experiments in Fluids Vol. 50, No. 1, January 2011 
${ }^{2}$ Louis J. Souverein, Pierre Dupont, Jean-François Debiève and Jean-Paul Dussauge, "Effect of Interaction Strength on Unsteadiness in Turbulent Shock-Wave-Induced Separations", AIAA Journal Vol. 48, No. 7, 2010

${ }^{3}$ S. Piponniau, J. P. Dussauge, J. F. Debiève \& P. Dupont, "A simple model for low-frequency unsteadiness in shock-induced separation", Journal of Fluid Mechanics Vol. 629, pp87-108, June 2009

${ }^{4}$ F. Moisy, "PIVMAT and ezyfit", http://www.fast.u-psud.fr/ moisy/index_en.php (Paris-Sud University)

${ }^{5}$ Damien Garcia "Robust smoothing of gridded data in one and higher dimensions with missing values", Computational Statistics and Data Analysis, 16 September 2009

${ }^{6}$ D. B. Helmer, L. M. Campo \& J. K. Eaton “Three-dimensional features of a Mach 2.1 shock/boundary layer interaction”, Experiments in Fluids Vol. 53, No.5, November 2012

${ }^{7}$ H. Babinsky, J. K. Harvey, "Shock Wave-Boundary layer interactions", Cambridge University Press, 2011

${ }^{8}$ Ethan Eagle, "An Experimental Study of Three-Dimensional Inlet Shock-Boundary Layer Interactions", Ph.D. Thesis (University of Michigan, Ann Arbor)

${ }^{9}$ Korkegi, R. H., "Comparison of Shock-Induced Two- and Three- Dimensional Incipient Turbulent Separation". AIAA J. 13 (4), 534-535. http://doi.aiaa.org/10.2514/3.49750, 1975

${ }^{10}$ Adamson, T.C. \& Messiter, A.F., "Analysis of two-dimensional interaction between shock waves and boundary Layers", Annu. Rev. Fluid Mech. 12, 103-138. DOI: 10.1146/annurev.fl.12.010180.000535, 1980

${ }^{11}$ Panaras, A., "Review of the Physics of Swept-Shock/Boundary Layer Interactions", Prog.in Aerospace Sci. 32,173244., 1996

12 Delery, J. \& Dussauge, J.P., "Some physical aspects of shock wave/boundary layer interactions", Shock Waves 19, 453468, 2009

${ }^{13}$ Alvi, F. S. \& Settles, G. S., "Physical Model of the Swept Shock Wave / Boundary-Layer Interaction Flow field", AIAA J. 30 (9), 2252-2258, 1992

${ }^{14}$ Bruce, P.J.K. \& Babinsky, H., "An experimental study of transonic shock/boundary layer interactions subject to downstream pressure perturbations”, Aerosp. Sci. Technol. 14 (2), 134-142, 2010

${ }^{15}$ Handa, T., Masuda, M. \& Matsuo, K., "Three-Dimensional Normal Shock-Wave /Boundary-Layer Interaction in a Rectangular Duct", AIAA J. 43, 2182-2187,2005

${ }^{16}$ Doerffer, P. \& Dallmann, U., "Reynolds number effect on separation structures at normal shock wave/turbulent boundarylayer interaction", AIAA J. 27, 1206-1212, 1989

${ }^{17}$ Humble, R.A., Elsinga, G.E., Scarano, F. \& Van Oudheusdin B.W., "Three-dimensional instantaneous structure of a shock wave/turbulent boundary layer interaction”, J. Fluid Mech. 622, 33-62, 2000

${ }^{18}$ Korkegi, R. H., "A simple correlation for incipient turbulent boundary layer separation due to skewed shock wave", AIAA J. 11, 1578-1579, 1974

${ }^{19}$ Amit Surana, Oliver Grunberg, \& George Haller, "An Exact Theory of Three-Dimensional Fixed Separation in Unsteady

Flows", Physics of Fluids, 20, 2008 